\title{
Rationalizing and Restricting Antibiotic Use by Utilizing a Proactive Justification Form
}

\author{
Banupriyanga Karuppiah $^{1} \cdot$ Suresh Chelliah ${ }^{1} \cdot$ Senguttuvan Duraisamy ${ }^{1}$
}

Received: 14 April 2021 / Accepted: 2 July 2021 / Published online: 7 August 2021

(c) Dr. K C Chaudhuri Foundation 2021

To the Editor: Around $75 \%$ of admitted children receive antibiotics in India, mostly unnecessarily or inappropriate, producing antibiotic resistance [1]. Antibiotic stewardship program (ASP), is a multidisciplinary programme that helps rationalize and restrict use of antibiotics [2]. Data on pediatric focused ASPs are rare and necessary because of unique indication, dosing, and vulnerability for lifetime resistance $[3,4]$.

In this quasi-experimental study, we evaluated the efficacy of an intervention, wherein the pediatricians had to fill a justification form before starting antibiotics.

Children who were admitted to the hospital from June 2018 to March 2019 were selected by systematic random sampling and enrolled in study as postintervention group. Children who were admitted to the pediatric ward previous year, during the same time period, i.e., June 2017 to March 2018 were taken as preintervention group.

Pediatricians had to fill the justification form within $24 \mathrm{~h}$ of starting antibiotic after cultures, that was notified within 48 to $72 \mathrm{~h}$. If antibiotics had to be continued, justification should be written in the form, and reviewed on day 7 or at discharge. Any decision to continue antibiotic further needed justification.

Similar pattern of illness was seen in both the groups. Of the post-intervention group, 55\% were started on antibiotics as against $82.4 \%$ in the preintervention group ( $p$ value $<0.001)$. De-escalation was done in $17.1 \%$ and $1.9 \%$ in the post- $\&$ preintervention group ( $p$ value $<0.001$ ). Duration of antibotics therapy was significantly lower in the postintervention group ( $p$ value $<0.001$ ).

Disease spectrum among the groups being comparable, the reduction in initial usage and de-escalation of antibiotics

Suresh Chelliah

chelliah.suresh@yahoo.co.in

1 Department of Pediatrics, Kauvery Hospital, Trichy,

Tamil Nadu 620001, India are attributed to the awareness created by the justification form. The written commitment was probably the most important factor in reducing antibiotic usage.

We conclude that implementation of antibiotic justification form is a simple and cost-effective intervention with vast implications in any clinical setup, thereby reducing antibiotic resistance.

\section{Declarations}

Conflict of Interest None.

\section{References}

1. Levy ER, Swami S, Dubois SG, Wendt R, Banerjee R. Rates and appropriateness of antimicrobial prescribing at an academic children's hospital, 2007-2010. Infect Control Hosp Epidemiol. 2012;33:346-53.

2. Chandy SJ, Michael JS, Veeraraghavan B, Abraham OC, Bachhav SS, Kshirsagar NA. ICMR programme on antibiotic stewardship, prevention of infection \& control (ASPIC). Indian J Med Res. 2014;139:226-30.

3. Smith et al. Inpatient antimicrobial stewardship in paediatrics: a systematic review. J Pediatr Infect Dis Soc. 2015;4:e127-e35.

4. Magsarili HK, Girotto JE, Bennett NJ, et al. Making a case for pediatric antimicrobial stewardship programs. Pharmacotherapy. 2015;35:1026-36.

Publisher's Note Springer Nature remains neutral with regard to jurisdictional claims in published maps and institutional affiliations. 